\title{
Chemical functionalization of polyethylene surfaces by plasma-assisted carbene
}

\section{insertion}

Zhen Hu* ${ }^{a, b}$ Mark G. Moloney*, ${ }^{*}$ Emily Parker, ${ }^{b}$ Shuyun Chng, ${ }^{b, c}$ Linda Y.L. $\mathrm{Wu}^{c}$

${ }^{a}$ School of Chemistry and Chemical Engineering, MIIT Key Laboratory of Critical Materials Technology for New Energy Conversion and Storage, Harbin Institute of Technology, Harbin 150001, China.

${ }^{b}$ Department of Chemistry, Chemistry Research Laboratory, University of Oxford, Oxford. OX1 3TA, UK

${ }^{c}$ Singapore Institute of Manufacturing Technology, 71 Nanyang Drive, Tower Block, Singapore 638075.

E-mail: mark.moloney@chem.ox.ac.uk (Mark G. Moloney); huzhen@ hit.edu.cn (Zhen Hu)

Tel : +44 (0)1865 275656; Fax : +44 (0)1865 275674

Abstract: Controlled surface modification of PE is achieved by an efficient and facile approach via plasma-assisted carbene insertion generated using diazo chemistry. The combination of plasma and carbene insertion shows a synergistic effect, which leads to an obvious enhancement of surface grafting, as shown qualitatively by a combination of IR and UV reflectance spectroscopy, and XPS analysis. The chemical modification may be observed spectroscopically, and the approach provides an opportunity for the convenient modification of low surface energy materials.

\section{Introduction}

Surface modification of materials can adjust surface characteristics without changing their bulk properties, and is an important route for the construction of functional materials; in particular, plasma treatment has been used to improve wetting and adhesion of polymers by introducing a variety of polar chemical groups.[1] Importantly, the plasma modifies only the uppermost layer (nanometer scale) of a polymer surface,[2-5] does not use solvents or generate chemical waste and causes less degradation and roughening of the material than chemical treatments,[6-9] and alteration of the discharge parameters (such as power, pressure, gas, etc.)[8] gives a flexible process for the introduction of a variety of functional groups onto a polymer surface.[10-14] However, this flexibility can be problematic, requiring careful multiparametric optimisation of the plasma process.[15] Plasma modification has proved to be of particular importance in low surface energy material such as polyethylene.[16, 17]

Compared to such physical activation, a wet chemical approach does not require specialized 
equipment and is more suitable for the treatment of porous 3-D substrates. Due to the low chemical reactivity of many polymer surfaces, however, surface modification often requires strong acid or alkali conditions[18-22] and these harsh reaction conditions often damage the surface and internal structure of polymers, leading to deterioration of mechanical and bulk properties. An alternative direct surface modification under milder conditions by using highly reactive diarylcarbenes, generated by thermolysis or photolysis of precursor diaryldiazomethanes,[23] has been developed, and been shown to modify the macroscopic properties of material surfaces[24, 25] for the introduction of a range of properties (e.g. visible[26] and fluorescent[27] chromophore, adhesion,[28, 29] biocidal,[30] and hydrophobicity[31, 32] effects) onto a variety of substrates. However, even in this process, the degree of introduced functionality is still low for low surface energy polymers such as polyethylene. Therefore, it was of interest to develop this carbene process to less reactive, low surface energy polymers such as polyethylene, and an indication that plasma modification might be useful in this regard came from earlier reports that this technique could be successfully applied to PE.[33-35] Since O-H bonds have better reactivity than C-H bonds towards carbene insertion processes, activation of the surface of a polymer by plasma-generated oxygenated functional groups (alcohol, carbonyl) would be expected to both increase the chemical reactivity of the polymer, but also its surface wettability, which would in turn improve the contact of the diaryldiazomethane modifying solution with the polymer surface. 




Scheme 1. The surface modification of PE by plasma assisted diazo chemistry

\section{Results and Discussion}

The surface treatment route of PE by plasma assisted diazo chemistry which was investigated is shown in Scheme 1. In order to achieve material modification, PE was first treated with oxygen plasma (Stage 1, Scheme) to achieve initial activation. This was then exposed to diarylcarbene; thus, dimethoxydiphenyldiazomethane (synthesis and characterization is given in the Supporting Information) dissolved in acetone was used to treat PE which after evaporation of solvent gave physisorbed material, which was thermolysed $\left(120^{\circ} \mathrm{C}\right)$ to initiate the carbene reaction (Stage 2, Scheme). In this process, collapse of the diaryldiazomethane (and its associated coloration) with loss of nitrogen gas, gave PE with yellow or pale yellow color, which comes from the chromophore of the introduced aromatic residues (Fig. 1). If this is done after plasma pretreatment PE is obtained which is darker in colour, consistent with a higher surface loading. This activated PE could then further treated with diazonium salts, leading to diazo coupling (Stage 3, Scheme), and giving even more intensely coloured materials, as a result of the introduction of an azo chromophore; this outcome gave direct visual indication of a successful surface modification 
sequence.

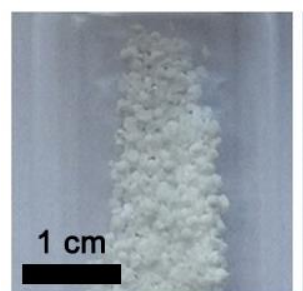

PE blank

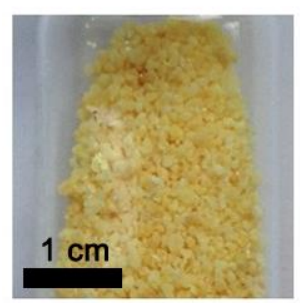

PE+plasma+diazo

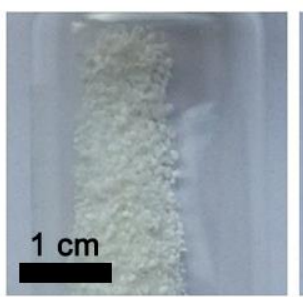

$\mathrm{PE}+$ plasma

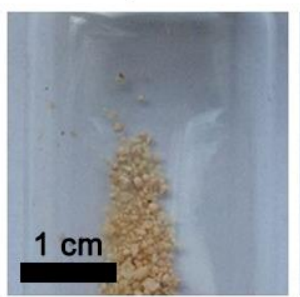

$\mathrm{PE}+$ plasma+diazo

+diazonium salt 1

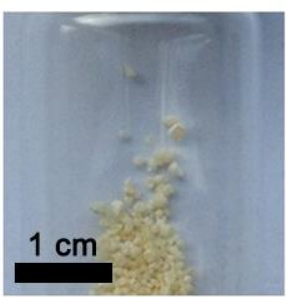

PE+diazo



+diazonium salt 2

Figure 1. The representative photographs of PE particles modified by plasma assisted diazo insertion chemistry. The PE samples are recorded as PE + plasma (treatment time 10, 20 or 30 $\min )+$ diazo + diazonium salt $\left(1 \mathrm{R}^{\prime}=-\mathrm{OH} ; 2 \mathrm{R}^{\prime}=-\mathrm{COOH}\right)$ as appropriate

In addition to this visual change, the successful functionalization of the PE was confirmed by several independent techniques. Firstly, attenuated total reflection-infrared spectroscopy (ATR-IR) was used; peaks were assigned by comparison to reported group frequency values.[36] In order to better compare the intensity of peaks, the data were normalized to the peak intensity at $\sim 2870 \mathrm{~cm}^{-1}$ (C-H stretching vibration, the strongest peak of PE polymer). As shown in Fig. 2a, all the samples show a noticeable absorption at $\sim 1471 \mathrm{~cm}^{-1}$ corresponding to the $\mathrm{C}-\mathrm{H}$ bending vibration of the PE. Without plasma pretreatment, the IR spectrum of PE which has been directly modified with the carbene does not show a significant difference when compared with pristine PE; this suggests that direct reaction of the carbene with the unactivated surface does not occur. However, after plasma-assisted diarylcarbene modification, new bands at $\sim 1507,1581$ and $\sim 1603 \mathrm{~cm}^{-1}$ were detected, consistent with the presence of the expected aromatic residues. As expected, the characteristic bands for aromatic ether bending at $\sim 1020 \mathrm{~cm}^{-1}$ (R-O) and $\sim 1250 \mathrm{~cm}^{-1}$ (Ar-O) were also present for modified PE. These IR results provide direct evidence for modification of the PE with the diarylcarbene. Moreover, it was also found that the plasma treatment time affects the degree of surface grafting. In particular, the IR spectrum of PE + plasma $20+$ diazo shows a relatively higher peak intensity of grafted diarylmethoxyl units, suggesting that 20 minute plasma pretreatment is the optimum. Shorter time plasma pretreatment did not give sufficient introduction 
of functional groups. To extend the range of the surface functional groups, the activated PE was further modified by diazonium coupling. As shown in Fig. 2b, the appearance of typical absorption peaks for $-\mathrm{N}=\mathrm{N}-$ at $\sim 1680 \mathrm{~cm}^{-1}$ provided evidence of the coupling reaction. Similarly, plasma pretreated PE shows a relatively high peak intensity of $-\mathrm{N}=\mathrm{N}-$ group, which indicates the plasma treatment enhanced the diazonium coupling effect.
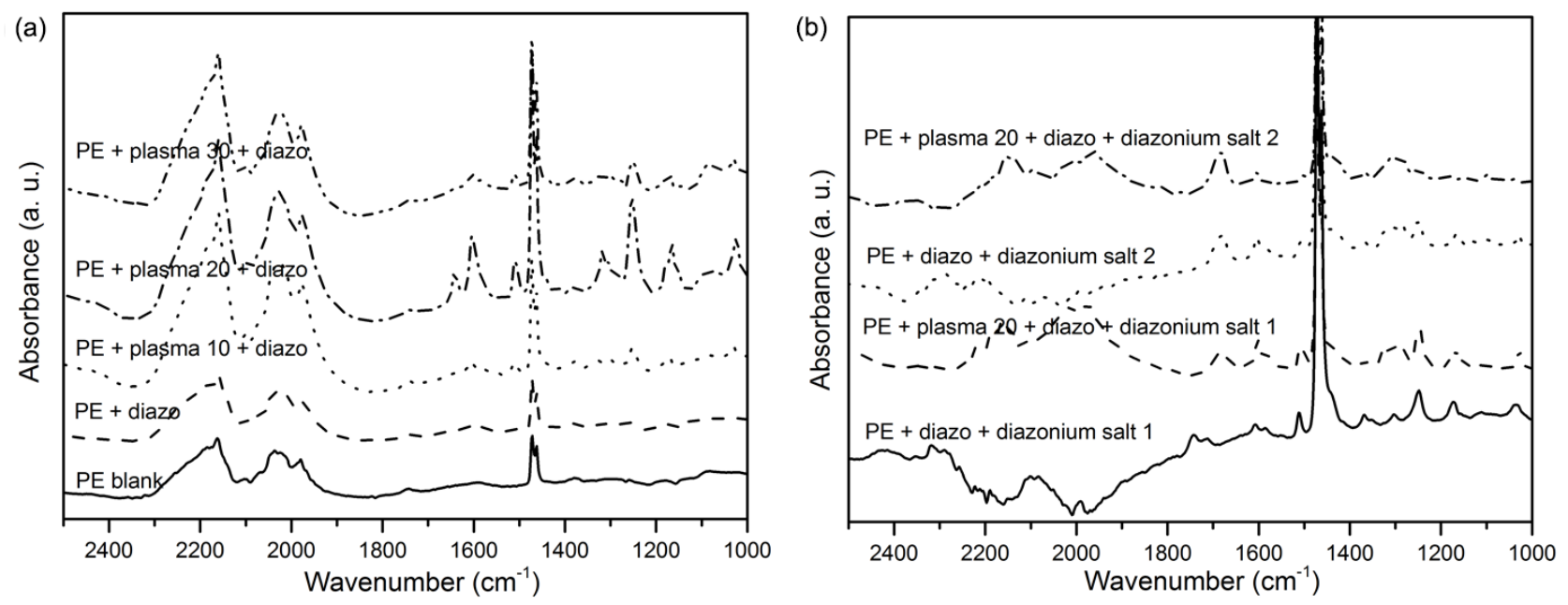

Figure 2. The ATR-IR spectra of modified PE via plasma assisted diazo chemistry (a) PE modified with/without plasma and diarylcarbene (Stages 1 and 2, Scheme 1); and (b) PE modified with/without plasma and diarylcarbene and diazonium salt (Stages 1, 2 and 3, Scheme 1).

The successful functionalization of PE was further confirmed by diffuse reflectance (solid state) $\mathrm{UV}-\mathrm{Vis}$ spectroscopic analysis, and the results are shown in Fig. 3; we have recently reported a correlation of solution and diffuse reflectance UV behavior using this technique.[37] Without modification, the pristine PE does not show an obvious UV absorption (Fig. 3a, PE blank). After diarylcarbene treatment (Scheme 1, Stage 2), a broad peak at $\sim 285-400 \mathrm{~nm}$ was detected in all samples, which can be attributed to the newly introduced aromatic rings (Fig. 3a and Scheme 1), but the intensity of absorption is significantly better for all samples which have been subjected to plasma treatment and the sample (PE + plasma $20+$ diazo) is strongest among all the samples. This result is consistent with the ATR-IR data, which indicates $20 \mathrm{~min}$ plasma treatment is most favorable for the attachment of the carbene. After diazonium coupling of the above samples (Scheme 1, Stage 3 and Fig. 3b), the absorption at $\sim 365 \mathrm{~nm}$ is greatly enhanced due to the grafting of $-\mathrm{N}=\mathrm{N}$ - groups. The envelope of absorption is broadly similar for all modified samples in Fig 3 , as would be expected, since the introduced chemical functional groups (azo, aromatic) are similar, although their intensity varies, as would be expected depending on the surface loading. For this 
reason, they show similar colour (Figure 1); however, their colour is not identical, and this is a result of absorbance differences in the spectrum in the visible region 450-600. It is clear that plasma pretreated samples present a stronger UV-visible absorption, providing solid indication of the synergistic effect between plasma and diazo chemistry.
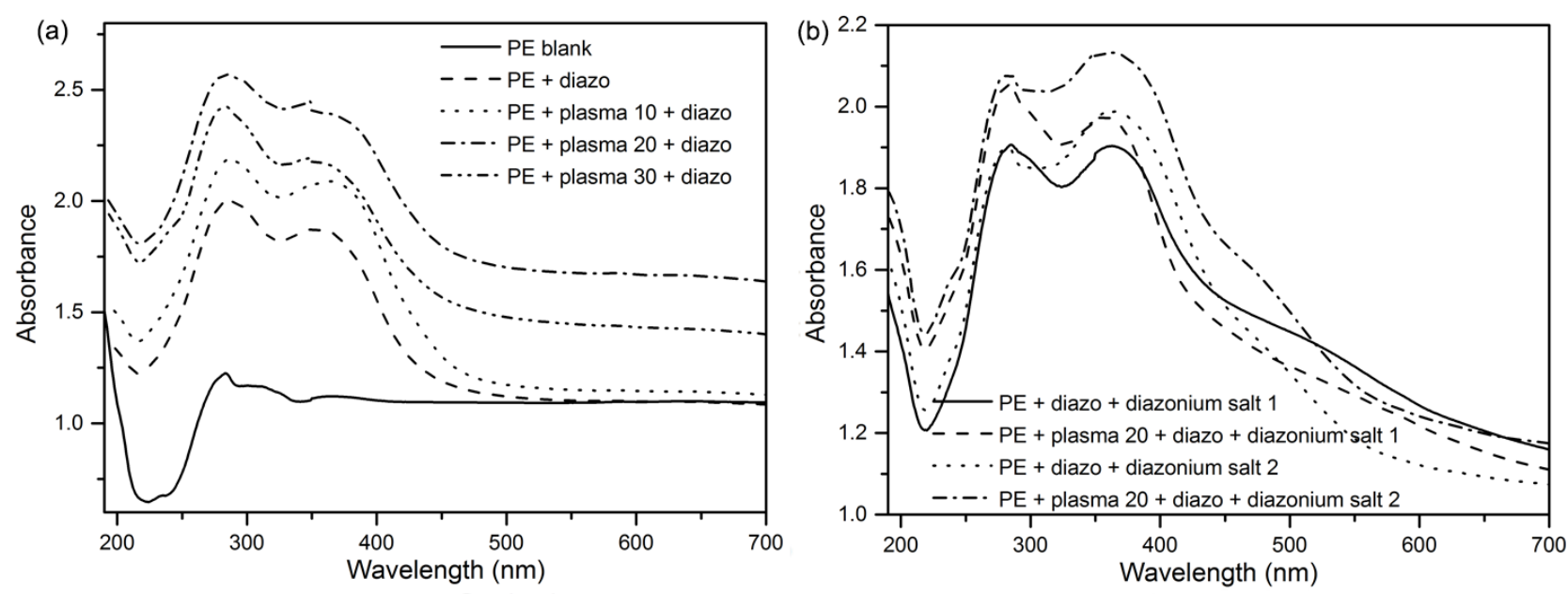

Figure 3. The solid state UV-Vis spectra of modified PE via plasma-assisted diazo chemistry (a) PE modified with/without plasma and diarylcarbene (Stages 1 and 2, Scheme 1); and (b) PE modified with/without plasma and diarylcarbene and diazonium salt (Stages 1, 2 and 3, Scheme 1). A = $\log (\mathrm{Io} / \mathrm{I})$, where Io is the intensity incident on the sample and I is the intensity that passed through it.

The surface chemical composition of modified PE was further examined by XPS analysis; observed Binding Energy values were assigned by comparison to standard database compilations.[38] Simple diarylcarbene treatment does not cause significant change of element mass ratio, and provides evidence of a low degree of functionality (Fig. 4b). The oxygen plasma treatment grafts various oxygen containing functional groups onto the PE surface, and as a result, the element mass ratio of oxygen increases from 7.0 to $29.3 \%$ (Fig. 4c). Quantitative analysis shows increase of oxygen mass ratio for the (PE + plasma20 + diazo) sample (Fig. 4d), and this may be attributed to the introduction of diarylcarbene groups. After the diazonium coupling (Fig. 4e, f), the XPS survey spectra revealed an obvious N1s peak, confirming the presence of $-\mathrm{N}=\mathrm{N}$ - groups on the PE surface. Furthermore, according to the element mass ratio for nitrogen, the density of functional groups $\left(\mathrm{R}^{\prime}=-\mathrm{OH}, \mathrm{R}^{\prime}=-\mathrm{COOH}\right)$ on the $(\mathrm{PE}+$ plasma20 + diazo + diazonium 1$)$ and $(\mathrm{PE}+$ plasma20 + diazo + diazonium 2) samples can be calculated to be $\sim 1.12$ and $\sim 1.82 \mathrm{mmol} / \mathrm{g}$, respectively. This value is much higher than those of previous results, $[17,21]$ and indicates the 
plasma assisted diazo chemistry is a very efficient modification method for a low surface energy polymer. In order to further prove the $-\mathrm{OH}$ group is successfully attached on the PE, the (PE + plasma20 + diazo + diazonium 1) was reacted with 2-bromoisobutyryl bromide; bromine was readily detectable by XPS analysis (Fig. S3).
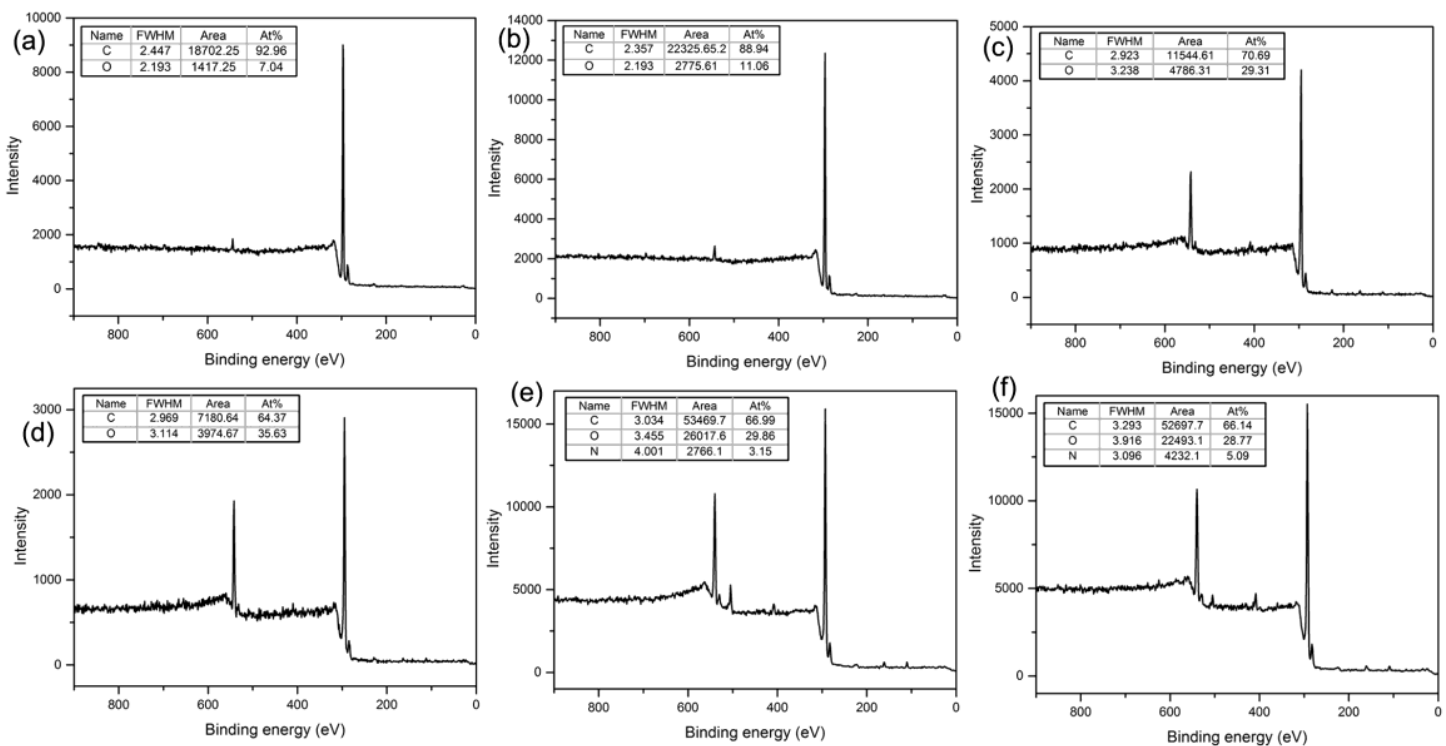

Figure 4. The XPS survey spectra of (a) PE blank, (b) PE + diazo, (c) PE + plasma, (d) PE + plasma20 + diazo, (e) $\mathrm{PE}+$ plasma20 + diazo + diazonium 1, (f) PE + plasma20 + diazo + diazonium 2.

The ATR-IR, UV-Vis and XPS results were further corroborated by ${ }^{13} \mathrm{C}$ NMR spectroscopic analysis (Fig. 5). Both samples present a prominent resonance at $\sim 30 \mathrm{ppm}$, due to the sp3 carbons of PE structure. After carbene modification, a new peak located at $~ 50 \mathrm{ppm}$ is observed, most likely corresponding to the methoxy carbons introduced by the diarylcarbene reaction. Moreover, the multiple peaks emerging at $\sim 110-140 \mathrm{ppm}$ can be attributed to carbons in the newly introduced benzene rings. In particular, the sample $(\mathrm{PE}+$ plasma $20+$ diazo + diazonium 1$)$ presents a small peak at $160 \mathrm{ppm}$, which may correspond to the carbons on the benzene ring that connecting with the $-\mathrm{OH}$ groups. Similarly, for $(\mathrm{PE}+$ plasma $20+$ diazo + diazonium 2), the small resonance located at $\sim 175 \mathrm{ppm}$ is attributed to the $-\mathrm{COOH}$ group grafted by diazonium coupling. 

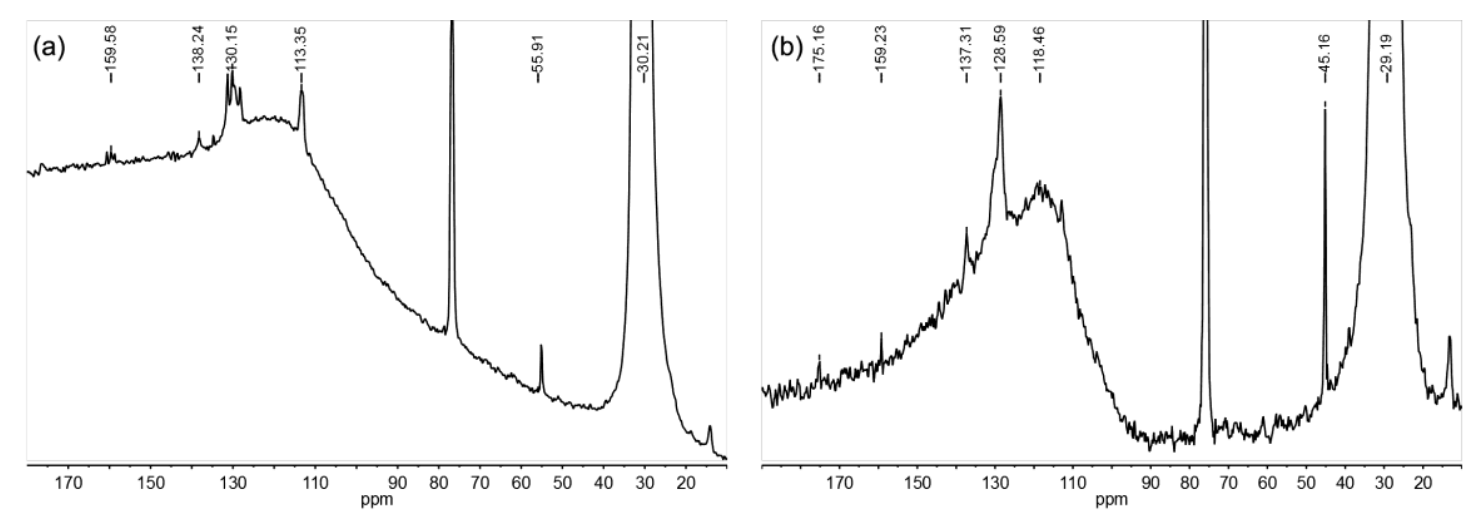

Figure 5. Solid state ${ }^{13}$ CNMR spectra of (a) (PE + plasma $20+$ diazo + diazonium 1), (b) (PE + plasma $20+$ diazo + diazonium 2).

\section{Conclusions}

PE particles could be readily modified using plasma-assisted diazo carbene and diazonium coupling chemistry, which introduces functional groups at the PE surface in a controlled manner. The combination of plasma and diazo chemistry shows an obvious synergistic effect by incorporating more reactive surface functionality which is capable of reacting with the carbene intermediate leading to covalent bond formation, although the long-term stability of this modification has not been assessed. The presence of the modified surface has been demonstrated qualitatively using a combination of IR, UV and NMR analysis, and quantitatively by XPS analysis, which suggests a surface loading of nitrogen of 1.1-1.8 $\mathrm{mmol} / \mathrm{g}$. This approach provides efficient modification of the surface properties of low surface energy materials using a simple process under mild conditions, which may find application for the design of new surface modified materials.

\section{Acknowledgements}

We thank the National Natural Science Foundation of China (no. 51673053), Key Technologies R \& D Program of Heilongjiang Province (no. GA14A101), and the Fundamental Research Funds for the Central Universities (no. HIT. IBRSEM. 2013016). The authors also thank Ashley Shepherd and Nicholas H. Rees for assistance with the XPS and solid state NMR analysis.

\section{References}

[1] A. Dinklage, T. Klinger, G. Marx, L. Schweikhard Plasma Physics, Springer, New York, 2005.

[2] H. Yasuda, Plasma for Modification of Polymers, J. Macromol. Sci., Part A: Pure Appl.Chem., 10 (1976) 383-420. 
[3] I.H. Coopes, K.J. Gifkins, Gas Plasma Treatment of Polymer Surfaces, J. Macromol. Sci., Part A: Chem., 17 (1982) 217-226.

[4] M.K. Shi, J. Christoud, Y. Holl, F. Clouet, Functionalization by Cold Plasmas of Polymer Model Surfaces (Hexatriacontane and Octadecyloctadecanoate) Studied by Contact Angle Measurements, XPS, and FTIR Spectroscopy, J. Macromol. Sci., Part A: Pure Appl.Chem., 30 (1993) 219-239.

[5] A. Mas, H. Jaaba, F. Schue, A.M. Belu, C. Kassis, R.W. Linton, J.M. Desimone, Poly(Hydroxybutyrate-co-9\% Hydroxyvalerate) Film Surface Modification by $\mathrm{Ar}, \mathrm{O} 2, \mathrm{H} 2, \mathrm{O} / \mathrm{O} 2, \mathrm{H} 2 \mathrm{O}$, and H2O2 Plasma Treatment, Journal of Macromolecular Science, Part A, 34 (1997) 67-79.

[6] S.R. Desai S, Surface modification of polyethylene, in: e. Albertsson AC (Ed.) Long-term properties of polyolefins, Springer, New York, 2004, pp. 231-293.

[7] C. Chan, T. Ko, H. Hiraoka, Polymer surface modification by plasmas and photons, Surf Sci Rep, 24 (1996) 3-54.

[8] J. Lane, D. Hourston, Surface treatments of polyolefins, Prog Org Coat, 21 (1993) 269-284.

[9] M. Ozdemir, C. Yurteri, H. Sadikoglu, Physical polymer surface modification methods and applications in food packaging polymers, Crit. Rev. Food Sci. Nutr., 39 (1999) 457-477.

[10] J. Kong, D. Lee, H. Kim, Surface modification of low density polyethylene (LDPE) film and improvement of adhesion between evaporated copper metal film and LDPE, J Appl Polym Sci, 82 (2001) 1677-1690.

[11] M. Aouinti , P. Bertrand, F. Poncin-Epaillard Characterization of polypropylene surface treated in a CO2 plasma, Plasmas Polym, 8 (2003) 225-236.

[12] N. Medard, J. Soutif, F. Poncin-Epaillard, Characterization of CO2 plasma-treated polyethylene surface bearing carboxylic groups, Surf Coat Technol, 160 (2002) 197-205.

[13] M. Wang, Y. Chang, F. Poncin-Epaillard, Acid and basic functionalities of nitrogen and carbon dioxide plasmatreated polystyrene, Surf Interface Anal, 37 (2005) 348-355.

[14] P. Wang, K. Tan, E. Kang, K. Neoh, Surface functionalization of low density polyethylene films with grafted poly(ethylene glycol) derivatives, J Mater Chem, 11 (2001) 2951-2957.

[15] J. Abenojar, R. Torregrosa-Coque, M.A. Martínez, J.M. Martín-Martínez, Surface modifications of polycarbonate (PC) and acrylonitrile butadiene styrene (ABS) copolymer by treatment with atmospheric plasma, Surface \& Coatings Technology, 203 (2009) 2173-2180.

[16] N. Patra, J. Hladik, L. Martinová, Investigating the thermal properties of polyethylene plasma modified by using unconventional chemical vapors, Journal of Thermal Analysis and Calorimetry, 117 (2014) 229-234.

[17] N. Patra, J. Hladik, M. Pavlatová, J. Militký, L. Martinová, Investigation of plasma-induced thermal, structural and wettability changes on low density polyethylene powder, Polymer Degradation and Stability, 98 (2013) 1489-1494.

[18] J.M. Goddard, J.H. Hotchkiss, Polymer surface modification for the attachment of bioactive compounds, Prog. Polym. Sci., 32 (2007) 698-725.

[19] J. Goddard, J. Talbert, J. Hotchkiss, Covalent attachment of lactase to low density polyethylene films, J Food Sci, 72 (2007) E36-41.

[20] G. Tao, A. Gong, J. Lu, H. Sue, D. Bergbreiter, Surface functionalized polypropylene: synthesis, characterization, and adhesion properties, Macromolecules, 34 (2001) 7672-7679.

[21] K. Holmberg, H. Hyden, Methods of Immobilization of Proteins to Polymethylmethacrylate, Prep Biochem, 15 (1985) 309-319.

[22] J. Rasmussen, E. Stedronsky, G. Whitesides, Introduction, modification, and characterization of functional groups on surface of low-density polyethylene film, J Am Chem Soc, 99 (1977) 4736-4745.

[23] P.J. Davis, L. Harris, A. Karim, A.L. Thompson, M. Gilpin, M.G. Moloney, M.J. Pound, C. Thompson, Substituted diaryldiazomethanes and diazofluorenes: structure, reactivity and stability, Tetrahedron Lett., 52 (2011) 1553.

[24] C.L. Bagwell, D.M.L. Leonard, J.-P. Griffiths, M.G. Moloney, N.J. Stratton, D.P. Travers, Post-Polymerization Modification of Materials using Diaryldiazomethanes: Changes to Surface Macroscopic Properties, Macromol. React. Eng., 8 (2014) 170-180. 
[25] D. Leonard, M.G. Moloney, C. Thompson, Chemical modification of materials by reaction with diaryl diazomethanes, Tetrahedron Lett., 50 (2009) 3499-3502.

[26] K. Awenat, P.J. Davis, M.G. Moloney, W. Ebenezer, A Chemical Method for the Surface Functionalisation of Polymers, Chem. Commun., (2005) 990 - 992.

[27] H. Wang, J.-P. Griffiths, R.G. Egdell, M.G. Moloney, J.S. Foord, Chemical Functionalization of Diamond Surfaces by Reaction with Diaryl Carbenes, Langmuir, 24 (2008) 862-868.

[28] G.W. Nelson, E.M. Parker, K. Singh, C.F. Blanford, M.G. Moloney, J.S. Foord, Surface characterization and in situ protein adsorption studies on a carbene-modified polymer surface, Langmuir, 31 (2015) 11086-11096.

[29] C. Choong, J.S. Foord, J.-P. Griffiths, E.M. Parker, B. Luo, M. Bora, M.G. Moloney, Post-polymerisation modification of surface chemical functionality and its effect on protein binding, New J. Chem., 36 (2012) 1187-1200.

[30] J.P. Griffiths, B. Maliha, M.G. Moloney, A.L. Thompson, I. Hussain, Surface Functional Polymers by Post-Polymerization Modification using Diarylcarbenes: Introduction, Release and Regeneration of Hydrogen Peroxide and Bactericidal Activity, Langmuir, 26 (2010) 14142-14153.

[31] C. Shepherd, E. Hadzifejzovic, F. Shkal, K. Jurkschat, J. Moghal, E.M. Parker, M. Sawangphruk, D.R. Slocombe, J.S. Foord, M.G. Moloney, New Routes to Functionalize Carbon Black for Polypropylene Nanocomposites, Langmuir, 32 (2016) 7917-7928.

[32] J.-P. Griffiths, D.M.L. Leonard, M.G. Moloney, N.J. Stratton, Control Of Wetting Behavior Using Post-Polymerization Modifications Of Surface Chemical Functionality, J. Mol. Eng. Mater., 1 (2012) 1250002.

[33] S. Yang, M.C. Gupta, Surface modification of polyethyleneterephthalate by an atmospheric-pressure plasma source, Surface \& Coatings Technology, 187 (2004) 172-176.

[34] D. Bandopadhay, A. Tarafdar, A.B. Panda, P. Pramanik, Surface modification of low-density polyethylene films by a novel solution base chemical process, Journal of Applied Polymer Science, 92 (2004) 3046-3051.

[35] M. Lehocký, H. Drnovská, B. Lapčíková, A.M. Barros-Timmons, T. Trindade, M. Zembala, L.r. Lapčík Jr, Plasma surface modification of polyethylene, Colloids and Surfaces A: Physicochemical and Engineering Aspects, 222 (2003) 125-131.

[36] J. Coates, Interpretation of Infrared Spectra, A Practical Approach, in: "Interpretation of Infrared Spectra, A Practical Approach" in Encyclopedia of Analytical Chemistry, John Wiley \& Sons, Ltd, 2006, pp. 1-23.

[37] S. Chng, E.M. Parker, J.-P. Griffiths, M.G. Moloney, L.Y.L. Wu, A study of diazonium couplings with aromatic nucleophiles both in solution and on a polymer surface, Applied Surface Science, 401 (2017) 181-189.

[38] NIST X-ray Photoelectron Spectroscopy Database, Version 4.1 (National Institute of Standards and Technology,

Gaithersburg, 2012); http://srdata.nist.gov/xps/. 